\title{
L'ANALYSE LONGITUDINALE DU DISCOURS SUR L'ENVIRONNEMENT DANS LE RAPPORT D'ENTREPRISE
}

\author{
Le cas Deutsche Post-DHL
}

\section{Marie-Pascale Senkel ${ }^{1}$}

On observe un intérêt croissant tant dans le monde académique que dans celui de l'entreprise pour les questions environnementales. Parallèlement, le besoin en informations sur ces questions augmente, comme le soulignent Adams et Frost (2004). Le rapport d'activité, un support traditionnel de divulgation des informations financières aux actionnaires, permet aussi aujourd'hui à de nombreuses entreprises de communiquer sur leurs actions environnementales.

Notre question centrale dans cette communication est la suivante : Dans quelle mesure l'information environnementale diffusée dans le rapport d'activité se modifie-t-elle?

Pour répondre à la question nous avons choisi de nous centrer sur un secteur particulier, celui de la prestation de services logistiques (PSL) et notamment sur le prestataire classé $n^{\circ} 1$ en

1 Marie-Pascale Senkel est maître de conférences en Sciences de Gestion - Département Gestion Logistique et Transport de l'IUT de Saint-Nazaire (Université de Nantes), rattachée au LEMNA (Laboratoire d'Economie et de Management de Nantes).

Recherches en communication, $\mathrm{n}^{\circ} 35$ (2011). 
France par le magazine professionnel Logistiques Magazine, DHL. DHL est aussi qualifié de « leader mondial de la logistique ». Notre recherche se fonde d'abord sur une analyse comparative de la structure des rapports entre 1998 et 2009 puis sur une analyse du discours présent dans ces rapports.

Nous aboutissons à la conclusion que le champ environnemental est une préoccupation de plus en plus importante pour Deutsche Post-DHL au moins si l'on en croit la place accordée à cette question dans les rapports de l'entreprise. L'analyse longitudinale permet de mettre en évidence comment les préoccupations sur le management durable ont évolué dans cette entreprise.

L'intérêt pour les questions autres que financières présentes dans les rapports d'activité des entreprises n'est pas nouveau. Lentz et Tschirgi (1963) étudiaient déjà la place prise par les questions éthiques dans les rapports d'activités d'une population de 219 entreprises américaines. Néanmoins, cet intérêt augmente tant dans le monde académique que dans celui de l'entreprise (privée comme publique). Lié à l'intérêt croissant pour les questions environnementales, le besoin en informations sur ces thèmes augmente, comme le soulignent Adams et Frost (2004). Le rapport d'activité, un support traditionnel de divulgation des informations financières aux actionnaires, permet aussi aujourd'hui aux entreprises de communiquer sur leurs actions environnementales, sociales ou sociétales. Ce rapport annuel d'activité comporte à la fois des informations requises par la loi et d'autres informations à propos desquelles l'entreprise choisit elle-même de communiquer.

De nombreux travaux académiques se sont d'ailleurs intéressés à la représentation que les entreprises font d'elles-mêmes dans ces rapports (Igalens, 2004 et 2006). Les travaux en la matière ont souvent tendance à comparer des rapports d'entreprises appartenant à un même secteur d'activité (Gendron, 2006) ou à des entreprises « comparables », par exemple celles du CAC-40 pour Igalens (2006). Ortiz Martinez et Crowthern (2008) choisissent un autre angle, en étudiant entre 1998 et 2003 les rapports rédigés par l'entreprise Shell. Cette démarche, l'étude longitudinale du rapport d'activité, avait été initiée par Hogner (1982) et reprise entre autre par Guthrie et Parker (1989). Igalens (2006) souligne d'ailleurs l'intérêt de ce type d'analyse.

Dans ce travail, nous avons opté pour une étude longitudinale en étudiant les informations environnementales diffusées dans le rapport d'activité de Deutsche Post-DHL depuis 1998. Mikol (2001, p. 3) définit 
l'information environnementale comme " ayant trait aux liens entre l'activité de l'entreprise et l'environnement, et aux actions de l'entreprise en faveur de la protection et de la remise en état de l'environnement $\gg$. Dans une première partie, nous proposons un panorama des champs théoriques mobilisés pour analyser la diffusion d'informations environnementales. Dans une seconde partie nous présentons le cadre de notre recherche. Puis dans une troisième et dernière partie, nous en donnons les principaux résultats.

\section{La diffusion d'informations environnementales : entre théorie des parties prenantes et théorie de la légitimité}

\section{Théorie des parties prenantes}

Freeman (1984) est à l'origine de la théorie des parties prenantes (ou théorie des stakeholders). Cette théorie "inscrit l'entreprise au cour d'un ensemble de relations avec des partenaires qui ne sont plus uniquement des shareholders (actionnaires), mais des acteurs intéressés par les activités et les décisions de l'entreprise 》 (Capron \& QuairelLanoizelée, 2004, p. 97). Le terme de partie prenante fait référence à « un individu ou un groupe qui peut affecter l'accomplissement des objectifs d'une organisation ou qui est affecté par l'accomplissement des objectifs d'une organisation » (traduit de Freeman, 1984, p. 46). L'organisation est dès lors vue comme placée au centre d'un réseau complexe de relations avec différents individus ou groupes d'individus.

La théorie des parties prenantes s'interroge sur qui sont les parties prenantes de l'entreprise. Le modèle de Wood (1991) permet de distinguer les parties prenantes primaires et les parties prenantes secondaires ce qui conduit à présenter l'entreprise comme insérée dans un environnement global, dans une vision systémique. Caroll (1991) définit clairement les parties prenantes : propriétaires, clients, employés, communauté dans laquelle s'insère l'entreprise, concurrents, fournisseurs, groupes d'activistes, la société en général et tout groupe ou personne concerné par les activités de l'entreprise.

Afin de comprendre les processus qui amène les entreprises à divulguer des informations relatives à leur responsabilité environnementale plusieurs types de modèle s'offrent aux chercheurs. Les uns sont des modèles économiques (Théorie de l'agence). La divulgation 
d'informations environnementales permet d'apporter de la valeur aux actionnaires. Les autres modèles sont comportementaux. Ils replacent l'entreprise dans son contexte social. Dowling et Pfeffer (1975, p. 122) affirment que « organizations seek to establish congruence between the social values associated with or implied by their activities and the norms of acceptable behavior in the larger social system of which they are a part. Insofar as these two value systems are congruent we can speak of organizational legitimacy ». La théorie de la légitimité est dès lors largement mobilisée et de nombreux travaux ont montré que la communication sociétale est utilisée par les entreprises comme un outil dans leur stratégie de légitimation de leurs actions (Oxibar, 2009).

\section{La théorie de la légitimité}

Selon la théorie institutionnelle, les organisations ne dépendent pas uniquement de ressources matérielles et informationnelles pour survivre mais doivent aussi assurer leur légitimité face à leur environnement stratégique (Powell \& DiMaggio, 1991). Wood (1991) intégrait d'ailleurs la légitimité comme grand principe de la responsabilité sociale de l'entreprise : la société accorde une légitimité à l'entreprise. Si celle-ci n'utilise pas son pouvoir économique dans un sens favorable à la société, elle risque de le perdre.

La légitimité est définie par Suchman (1995, p. 574) comme « $a$ generalized perception or assumption that the actions of an entity are desirable, proper, or appropriate within some socially constructed system of norms, values, beliefs, and definitions ». Suchman assure d'ailleurs que les entreprises peuvent construire leur légitimité par une communication fréquente et intense.

De nombreuses études se sont d'ailleurs intéressées à la compréhension de la diffusion d'informations sociales et/ou environnementales vue comme un moyen de légitimer les activités des entreprises, notamment auprès de leurs parties prenantes (Deegan \& Gordon, 1996 ; Wilmshurst \& Frost, 2000 ; Campbell, 2003).

En synthèse, nous pouvons prendre à notre compte les apports de Swanson (1995) qui met en évidence trois grandes motivations à l'intégration d'actions sociales et/ou environnementales par l'entreprise et les appliquer à la diffusion d'informations environnementales :

- pour atteindre des objectifs en termes de profitabilité, retour sur investissement, volume de ventes. Il s'agit dans ce cas d'une communication orientée sur la performance économique, 
- pour répondre aux attentes des parties prenantes en légitimant l'action de l'entreprise auprès de ces dernières. Il s'agit alors d'une communication orientée vers les parties prenantes,

- pour affirmer ces dimensions dans l'identité même de l'entreprise. Il s'agit alors d'une communication motivée par les valeurs de l'entreprise.

Ces trois grandes motivations sont d'ailleurs utilisées dans une étude menée par Maignan et Ralston (2002) dans laquelle ces auteurs comparent le contenu de la communication environnementale et sociétale de 400 entreprises (anglaises, françaises, néerlandaises et américaines).

Trois questions principales vont structurer notre recherche :

- QR1 : Dans quelle mesure la diffusion d'informations sur les pratiques environnementales de l'entreprise Deutsche Post-DHL présentée dans ses rapports d'activité a-t-elle évoluée sur la période 1998-2009?

- QR2 : Quels sont les déterminants principaux à cette démarche de diffusion?

- QR3 : Quelle est la motivation principale exprimée par l'entreprise pour expliquer ses choix de communiquer sur ce thème?

\section{Méthodologie}

\section{Le choix du terrain de recherche}

Pour répondre à ces questions nous avons choisi de nous centrer sur un secteur particulier, celui de la prestation de services logistiques (PSL). Ce secteur est très sensible aux problématiques environnementales. Jupe (2005) classe le secteur des Transports parmi les secteurs sensibles aux questions environnementales ainsi que Oxibar (2009) qui affirme que ce secteur a "une visibilité environnementale due à des produits dont la consommation peut entraîner une atteinte aux ressources naturelles ».

Notre recherche se focalise sur DHL, le prestataire logistique classé $\mathrm{n}^{\circ} 1$ en France par le magazine professionnel Logistiques Magazine. DHL est aussi qualifié par ce magazine de «leader mondial de la logistique ».

Nous avons décidé de travailler sur les rapports d'activité annuels de la période 1998-2009. Pourquoi le choix de cette période ? La société 
DHL est fondée en 1969 par Adrian Dalsey, Larry Hillblom et Robert Lynn à San Francisco pour transporter par avion des documents entre San-Francisco et Honolulu. C'est à partir de 1998, que Deutsche Post AG commence à prendre des participations dans cette entreprise. En 2001, la participation devient majoritaire et en 2002, DHL devient une filiale de Deusche Post World Net. Le dernier rapport présent sur le site internet de Deutsche Post-DHL au moment de la conduite de l'étude est celui de 2009.

\section{La méthode de recherche}

La première étape fut la collecte des rapports d'activité au format pdf sur le site institutionnel de Deutsche Post-DHL. Les rapports d'activité depuis 1997 y sont présents ainsi que les rapports annexes, environnementaux et sociaux.

Le choix de l'unité d'analyse est un problème souvent évoqué dans les travaux antérieurs. Est-ce la phrase (Gray et al., 1995), le mot comme chez Deegan et Gordon (1996) ou encore l'équivalent-nombre de pages comme chez Oxibar (2005). Compte tenu de la taille de notre population nous avons souhaité minorer le risque d'erreur en retenant l'unité d'analyse la plus petite : le mot. En choisissant le mot comme unité d'analyse, nous évitons la subjectivité du codeur et nous obtenons un comptage qui est reproductible. De plus, une phrase comme celle-ci «We aim to reduce our use of fossil fuels, lower $\mathrm{CO} 2$ emissions and by 2012 to cut greenhouse gas emissions from our road transport activites in Europe by 5\% compared with the 1990 level » contient plusieurs informations relatives à la politique environnementale de l'entreprise.

Nous sommes aussi conscients de certaines faiblesses de cette unité d'analyse : le mot ne prend pas en compte des éléments non-narratifs comme les photos ou les graphiques comme le souligne Unerman (2000). Sorti de son contexte (la phrase), le mot peut perdre en signification (Milne \& Adler, 1999). Ainsi le mot environnement peut signifier l'environnement stratégique de l'entreprise ou son environnement naturel selon le contexte de la phrase. Nous avons donc replacé à chaque fois le terme recherché dans son contexte afin d'en appréhender la signification.

Les mots-clés recherchés dans le texte des rapports d'activité découlent du cadre proposé par la Global Reporting Intitiative (GRI). Lorsque qu'une information environnementale est présente dans le rapport d'activité, nous l'avons codée en nous appuyant sur le travail de 
Maignan et Ralston (2002) en cherchant d'abord à déterminer si cette information est intégrée aux valeurs de l'entreprise, si elle relève plutôt d'un principe de performance économique ou d'un principe de légitimité.

\begin{tabular}{|l|l|l|}
\hline \multicolumn{1}{|c|}{ Principe } & \multicolumn{1}{|c|}{ Définition } & $\begin{array}{l}\text { Exemple extrait d'un rapport } \\
\text { annuel Deutsche Post-DHL }\end{array}$ \\
\hline $\begin{array}{l}\text { Valeur de } \\
\text { l'entreprise }\end{array}$ & $\begin{array}{l}\text { La Responsabilité Sociale de } \\
\text { l'Entreprise (RSE) est présentée } \\
\text { comme un élément de la culture } \\
\text { de l'entreprise, comme une } \\
\text { valeur forte de l'entreprise. }\end{array}$ & $\begin{array}{l}\text { «As a responsible logistics } \\
\text { company, we consider mini- } \\
\text { mizing the adverse effects of our } \\
\text { own activities to be one of our } \\
\text { most important duties » }\end{array}$ \\
\hline $\begin{array}{l}\text { Performance } \\
\text { économique }\end{array}$ & $\begin{array}{l}\text { La RSE est présentée comme } \\
\text { un élément de la performance } \\
\text { économique de l'entreprise, de } \\
\text { sa compétitivité. }\end{array}$ & $\begin{array}{l}\text { «By the end of 2002, our parcel } \\
\text { Intercity Project, in collabora- } \\
\text { tion with Deutsche Bahn, should } \\
\text { result in 100 000 fewer truck } \\
\text { journey every year through the } \\
\text { use of rail transport » }\end{array}$ \\
\hline $\begin{array}{l}\text { Légitimité face } \\
\text { aux parties } \\
\text { prenantes }\end{array}$ & $\begin{array}{l}\text { La RSE est présentée comme } \\
\text { une réponse à la pression d'une } \\
\text { ou plusieurs parties prenantes. }\end{array}$ & $\begin{array}{l}\text { "Demand is now emerging for } \\
\text { climate-neutral products » }\end{array}$ \\
\hline
\end{tabular}

Tableau 1 : Codage de l'information sur les principes auxquels répond l'information environnementale

\section{Présentation et analyse des résultats}

La structure générale des rapports est relativement stable d'une période à l'autre, avec néanmoins quelques ruptures concernant le thème qui nous intéresse. C'est en 2001, qu'apparaît pour la première fois le terme "environment » dans le sommaire du rapport d'activité. Il est rapidement remplacé par une vision plus large, dépassant la seule notion de l'environnement ("safeguarding the future » puis «Sustainability »), pour finalement être inclus en 2007 dans un paragraphe sur les indicateurs non-financiers de la performance de l'entreprise.

Chaque année, le rapport d'activité a un titre essayant de mettre en valeur les tendances fortes de l'entreprise : construire un groupe mondial, montrer son intérêt pour les clients, montrer que malgré sa taille l'entreprise peut agir localement.... Aucun titre de rapport ne laisse apparaître la prise en compte de la dimension environnementale par l'entreprise. La volonté stratégique de devenir le $n^{\circ} 1$ mondial de 
la prestation de services logistiques est, elle, clairement affichée dès 1999. Pour reprendre le terme de de Serres et al. (2006), on observe une volonté de « Leadership » affirmée depuis 2007 : «As the world's largest logistics service provider ", "As the largest company in our industry $\gg .$. qui est le reflet de la stratégie menée par le groupe Deutsche Post depuis 1998 pour ne plus être seulement une entreprise de distribution de courrier mais devenir le numéro 1 de la prestation de services logistiques dans le monde comme le rappellent les titres des rapports d'activité de 1999 et de 2001 (« Deutsche Post World Net on its way to becoming $n^{\circ} 1$ worldwide »; «Integrated worldwide logistics is our future »).

Le titre du rapport 2009 et surtout sa déclinaison « By respecting people, the environment and our society, we are delivering results for everyone » marque un tournant dans la communication sur la dimension environnementale et sociale de l'entreprise puisque c'est la première fois que le terme " environment » apparaît dans un titre de rapport d'activité. Ceci est en cohérence avec le plan stratégique ("Strategy 2015 ») dévoilé la même année par le président Frank Appel. Ce plan stratégique a comme mot d'ordre « living responsibility ». Après avoir « assis » sa position de leader dans son domaine, le groupe Deutsche Post-DHL cherche ainsi à assurer sa légitimité auprès de ses différentes parties prenantes.

\section{Qualification de l'information environnementale présente dans les rapports d'activité}

Cette étape consiste à relever pour ensuite classer et organiser les informations à caractère environnemental qui sont diffusées dans le rapport d'activité. Sont considérées comme faisant partie de l'environnement les informations qui relèvent d'un des domaines suivants : recyclage, consommation d'énergie, biodiversité, gestion de l'eau, émissions, effluents, déchets, impacts environnementaux, dépenses et investissements en matière de protection environnementale... Ces différents mots clés ou thèmes clés font directement référence aux différents volets environnementaux de la ligne directrice pour le reporting environnemental et social proposés par la Global Reporting Initiative (GRI).

Précisons tout d'abord, que même si elle n'est pas dans le champ du travail présenté ici, l'information la plus largement diffusée concerne, 
non pas la dimension environnementale, mais la dimension sociale (informations relatives aux salariés).

\section{Quantité d'informations diffusée}

Pour chaque partie du rapport d'activité clairement identifiée comme relative à la dimension environnementale de la responsabilité sociale de l'entreprise, nous avons comptabilisé le nombre de mots, en référence aux travaux antérieurs qui établissent un lien positif entre le volume d'informations diffusé et l'importance accordée par l'entreprise au thème (Neu et al. 1998).

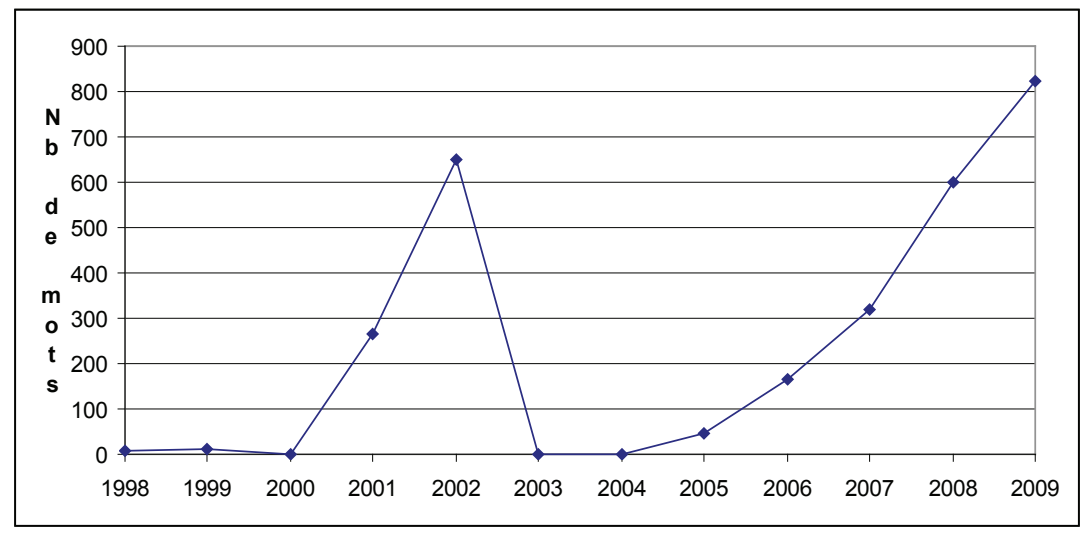

Graphique 1: Nombre de mots dans la partie Environnement du rapport d'activité

Le nombre de mots relatifs à la politique environnementale de l'entreprise est en moyenne de 240 mots sur la période 1998-2009, avec un écart-type de 297 mots. On note que de 1998 à 2000, la présence d'informations sur la politique environnementale est faible, voire nulle. Le volume d'informations environnementales commence à augmenter à partir de 2001 pour atteindre un pic en 2002. Dès 2003, l'information environnementale est à nouveau inexistante. C'est la date à laquelle l'entreprise édite son premier rapport indépendant relatif à l'environnement. Le rapport d'activité sert alors uniquement à donner cette information et à renvoyer le lecteur vers le rapport indépendant ou le site Internet de l'entreprise. A partir de 2005, même si l'entreprise continue d'éditer des rapports indépendants, les informations relatives à l'environnement dans le rapport d'activité ne cessent d'augmenter pour dépasser, en 2009, le pic atteint en 2002. 
Campbell (2003) mène une analyse longitudinale sur la divulgation d'informations environnementales par 100 entreprises britanniques entre 1974 et 2000 à partir des informations fournies dans les rapports d'activité. Son étude montre qu'en 2000, le nombre moyen de mots est proche de 750. En accord avec les résultats d'Oxibar (2003), nous trouvons que le volume d'informations environnementales dans les rapports de l'entreprise DHL est relativement faible. Plus récemment, Jenkins et Yakovleva (2006) constatent que : " although the level of social and environmental disclosure has increased over the years, companies still provide relatively little detailed social and environmental information in their Annual Reports... ».

\section{Forme des informations divulguées}

Comme le souligne Oxibar (2009), les informations divulguées peuvent revêtir une variété de formes (informations littérales, informations quantitatives qui peuvent être sous la forme d'un nombre, d'un pourcentage, d'une valeur monétaire, d'un rang...). L'information peut également être présentée sous la forme d'un tableau ou d'un graphique voire d'une photographie.

L'information environnementale contenue dans le rapport d'activité est principalement qualitative.

\begin{tabular}{|l|c|c|c|c|c|c|c|c|c|c|c|c|}
\hline & 98 & 99 & 00 & 01 & 02 & 03 & 04 & 05 & 06 & 07 & 08 & 09 \\
\hline $\begin{array}{l}\text { Information sous } \\
\text { forme littérale }\end{array}$ & 1 & 1 & & 1 & 1 & & & 2 & 7 & 2 & 5 & 15 \\
\hline $\begin{array}{l}\text { Information sous } \\
\text { forme qualitative }\end{array}$ & 0 & 0 & & 1 & 1 & & & 0 & 2 & 3 & 0 & 8 \\
\hline
\end{tabular}

Tableau 2 : Forme de l'information environnementale diffusée (nombre d'occurrences)

Lorsqu'elle est quantitative, cette information est majoritairement un nombre, dans des cas plus rare un pourcentage. A partir de 2006, l'entreprise fournit des informations chiffrées (nombre de véhicules utilisant le gaz naturel, nombre de tonnes de $\mathrm{CO}_{2}$ émis...) mais aucune information à caractère monétaire n'est fournie. Enfin, il s'agit principalement d'une information à caractère " positif » qui est divulguée puisque des termes comme «noise, congestion, incident, pollution... » n'ont aucune occurrence. 
Ces résultats sont conformes à ceux des travaux antérieurs, et notamment Oxibar (2003) qui montre que la forme privilégiée de divulgation d'informations environnementales dans le rapport d'activité est qualitative et descriptive. Ceci tend à montrer que les entreprises et notamment Deutsche Post-DHL restent très prudentes dans leur diffusion d'informations, ne fournissant aucune (ou peu de) données chiffrées qui pourraient être vérifiées par les parties prenantes et éventuellement faire l'objet de procédures judiciaires.

\section{Présentation de l'information environnementale}

C'est en 2001 que pour la première fois l'entreprise consacre une partie de son rapport d'activité à l'environnement (256 mots). C'est une « lettre d'intention » dans laquelle l'entreprise affiche sa volonté de travailler à l'avenir sur ce sujet : we aim/these aims/we hope. Ces verbes sont encore présents dans le document de 2006 ( « we aim to reduce our use of fossil fuels »). A partir de 2007, les verbes utilisés sont plus actifs «we are able to », " we take our responsibility », « we have four main positions »... En 2009, les declarations de l'entreprise sont pro-actives : «we are calling for the development of international (...) standards for measuring carbon (...) we are calling on governments and institutions to incentivise investment in carbon-efficient solutions... ». Elle montre ainsi sa volonté d'influer sur son environnement. Un effet d'annonce des mesures qui seront prises l'année suivante donne un sentiment de dynamisme ou de réponse par anticipation à des critiques potentielles de certaines parties prenantes ( « we hope to produce a separate environmental report for the first time in 2003 ; In 2006, we intend publication of a comprehensive Sustainability report... »).

Nous retrouvons dans le cas de l'entreprise DHL la même évolution dans la diffusion de l'information environnementale que celle mise en évidence par de Serres et al. (2006) dans leur étude sur le secteur bancaire. La diffusion d'information environnementale a commencé par une inclusion dans le rapport d'activité. L'entreprise a, à partir de 2003, réalisé un rapport environnemental indépendant. De 2006 à 2008, le 
rapport indépendant est devenu « Rapport de développement durable », incluant les dimensions environnementales, sociales, sociétales et économiques. En 2009, le rapport s'est basé sur les recommandations de la GRI. Il devient à partir de 2010 un rapport de « Responsabilité de l'entreprise $»$.

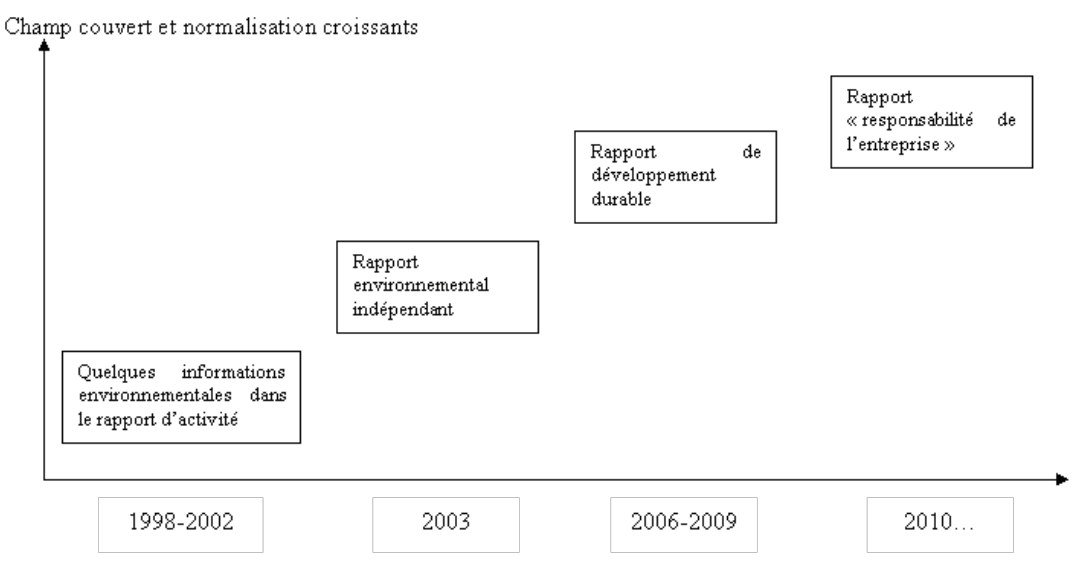

Source : Adapté de de Serres et al. (2006)

Figure 1 : Evolution de la divulgation d'information environnementale

On note une implication croissante de l'entreprise dans sa volonté de diffuser des informations environnementales et sociales et l'institutionnalisation de cette diffusion, notamment par la certification des rapports dédiés par des organismes indépendants et le recours à la forme de présentation suggérée par la grille de la GRI.

\section{Les parties prenantes évoquées}

Les parties prenantes les plus fréquemment citées dans le rapport d'activité (en moyenne sur la période) sont les clients, le personnel, les actionnaires, le gouvernement et enfin les fournisseurs.

Selon Oxibar $(2009$, p. 35) : «la communication sociétale apparaît ainsi comme une reddition de comptes sociaux à un ensemble d'individus ou groupes d'individus que les études tentent d'identifier ou dont elles cherchent à connaitre les besoins d'information ou les vecteurs les plus adaptés pour les atteindre ». Ainsi Internet est largement utilisé comme un complément à l'information environnementale diffusée dans le rapport d'activité, par des renvois pour obtenir plus 
de détails. De nombreuses études ont déjà montré que l'information diffusée sur Internet est en effet plus facilement actualisable. A partir de 2010 (annonce faite dans le rapport d'activité de 2009), le rapport indépendant ne sera disponible que sur Internet dans une version on-line. Il semblerait donc que pour l'entreprise Deutsche Post-DHL, le meilleur moyen de toucher les parties prenantes intéressées par les informations environnementales, sociales et sociétales soit de diffuser ces informations sur son site Internet. Notons que déjà en 2003 et 2004, l'information présente dans la partie environnementale du rapport d'activité ne servait qu'à renvoyer le lecteur vers le rapport indépendant ou vers le site Internet de l'entreprise.

\section{Quelles valeurs?}

Sur la période étudiée, Deutsche Post-DHL communique plutôt sur ses actions en matière environnementale en les présentant comme partie intégrante des valeurs de l'entreprise (« We have developped GoGreen as a group-wide program »; " As the largest company in our industry we take our environmental and social responsibility seriously... »). Nos résultats sont en désaccord avec ceux de Maignan et Ralston (2002) qui mettent en évidence dans leur échantillon que ce sont plutôt les entreprises américaines qui ont cette attitude. Maignan et Ralston (2002) montrent que les entreprises européennes de leur échantillon communiquent sur leur responsabilité sociale et environnementale en réponse à la pression des parties prenantes (48\% des entreprises européennes de leur échantillon contre 16\% des entreprises américaines). Doit-on y voir l'influence de DHL (entreprise américaine) dans la politique de Deutsche Post-DHL ? Cependant, sur les sept membres de direction $\mathrm{du}$ groupe quatre sont d'origine allemande et trois d'origine anglosaxonne. Doit-on y voir l'influence de la dimension internationale de ce groupe qui veut clairement se positionner comme leader mondial dans son secteur? Des études complémentaires sont nécessaires pour répondre à cette question. Cependant, même si l'information en matière environnementale devient «normée » comme nous l'avons montré au paragraphe 3.1.3, il est intéressant de noter que les entreprises gardent une part de «liberté » dans leur communication.

Une autre phénomène méritera d'être étudier sur le long terme, à savoir la présence simultanée dans le discours de Deutsche Post-DHL depuis 2008 de chacun des trois principes dans le discours de l'entreprise : l'information environnementale y est présentée à la fois comme 
une valeur de l'entreprise, comme permettant de légitimer son action face à ses parties prenantes (voire de demander l'aide de certaines parties prenantes secondaires) mais aussi comme un élément permettant d'assurer la performance de l'entreprise. Est-ce dans l'intégration de ces trois principes que vont communiquer à l'avenir les entreprises de taille internationale afin de répondre aux attentes de chacun des environnements dans lesquels elles agissent?

\section{Quelles pratiques environnementales?}

Les actions présentées dans le rapport d'activité concernent principalement :

- La multimodalité par le passage d'une partie du fret routier vers le transport ferroviaire (Rapports de 1999 et 2001),

- Des mesures répondant à la réglementation dans les rapports de 2001, 2002 (réglementation en matière de normes d'émissions des moteurs thermiques, réglementation en matière de bruit...),

- La certification ISO 14001, le management environnemental et la formation des salariés et leur sensibilisation aux problèmes environnementaux (Rapports de 2002 et 2007),

- Des mesures permettant d'atteindre les standards du protocole de Kyoto, à savoir la réduction des émissions totales des pays développés d'au moins 5\% sur la période 2008-2012 par rapport aux niveaux de 1990 (Rapport de 2006, 63).

La majorité des mesures présentées dans le rapport d'activité visent à réduire l'empreinte carbone de l'entreprise par le transfert de fret vers des modes moins émetteurs, par l'utilisation de moyens de transport plus sobres... Nous n'avons repéré aucun exemple d'initiatives (en collaboration avec un industriel ou un distributeur) dans laquelle Deutsche Post-DHL a pu mettre en place une nouvelle organisation logistique. Les exemples donnés visent principalement à « faire mieux » mais pas à « faire autrement ». Cela est particulièrement évident dans le texte du rapport de 2008 au sujet du programme GoGreen : « Using energy-efficient processes and technologies, employees who are environmentally conscious, climate-neutral products, using standardised systems ». 


\section{Conclusion}

Ce travail de recherche permet de dresser un tableau de l'évolution de la diffusion d'informations environnementales dans le rapport d'activité de Deutsche Post-DHL entre 1998 et 2009.

Nous aboutissons à la conclusion que le champ environnemental est une préoccupation de plus en plus importante pour Deutsche Post-DHL au moins si l'on en croit la place accordée à cette question dans les rapports de l'entreprise. Nous aboutissons également à la conclusion que l'entreprise a de plus en plus à cœur d'assurer sa légitimité et la confiance des parties prenantes par le biais de ces rapports en avançant le contrôle effectué par des tiers, le respect de normes (Iso 14001) et des recommandations de la GRI. L'analyse longitudinale permet de mettre en évidence comment les préoccupations sur le management durable ont évolué dans cette entreprise. En effet, des innovations technologiques importantes ont été réalisées dans le secteur des transports entre les années 1980 et 2000. Il reste à ce jour à innover dans le domaine des carburants ou de la technologie des moteurs. Selon nous, le domaine le plus demandeur d'innovation est cependant sans conteste le domaine organisationnel : rédaction des contrats, report modal, repenser les localisations, repenser les relations. Or jusqu'à aujourd'hui peu de choses concrètes ont été faites dans ce domaine si l'on en juge par la communication de DHL.

Si le rapport d'activité est une source souvent utilisée dans les travaux antérieurs (Tilt, 1994 ; Gray et al., 1995...), les entreprises accordent une place croissante à la publication d'un rapport indépendant. Comme le notent Igalens (2006) et de Serres et al. (2006), depuis la fin des années 90 , les rapports de responsabilité sociale indépendants du rapport d'activité annuel de l'entreprise se développent. Ces rapports indépendants n'apparaissent dans le cas de Deutsche Post-DHL qu'à partir de 2003. Une suite à cette recherche sera l'étude des informations diffusées dans ces rapports indépendants ainsi qu'une analyse comparative comme le propose Oxibar (2003) entre l'information diffusée sur Internet, dans les rapports d'activité et dans les rapports indépendants. 


\section{Références}

Adams, C.A. \& Frost, G. R. (2004). The development of corporate web-sites and implications for ethical, social and environmental reporting through these media. Edinburgh : The Institut of Chartered Accountants of Scotland.

Campbell, D. (2003). Intra- and intersectorial effects in environmental disclosures: evidences for the legitimacy theory ?. Business Strategy and the Environment, 12, 357-371.

Capron, M. \& Quairel-Lanoizelée, F. (2004). Mythes et réalités de l'entreprise responsable. Paris : La Découverte.

Caroll, A. B. (1991). The pyramid of corporate social responsibility: toward the moral management of organizational stakeholders. Business Horizons, July-August, 3948.

de Serres, A., Gendron, C. \& Ramboarisata, L. (2006). Etudes des pratiques des banques canadiennes en matière de divulgation d'information sur leur responsabilité sociale. Congrès annuel de l'Association des sciences administratives du Canada, Banff.

Deegan, C. \& Gordon, B. (1996). A study of the environmental disclosures practices of Australian corporations. Accounting and Business Research, 26 (3), 187-199.

Dowling, J. \& Pfeffer, J. (1975). Organizational legitimacy: social values and organizational behaviour. The Pacific Sociological Review, 18 (1), 122-136.

Freeman, R. (1984). Strategic management: a stakeholder approach. Marshfield : Pitman Publishing Luc.

Gendron, C. (2006). Les rapports de responsabilité sociale et de développement durable des entreprises financières d'économie sociale. Une analyse préliminaire. Cahier de la Chaire de responsabilité sociale et de développement durable, Montréal : École des sciences de la gestion, UQÀM, 10-2006.

Gray, R., Kouhy, R. \& Lavers, S. (1995). Methodological themes: constructing a research database of social and environmental reporting by UK companies. Accounting, Auditing and Accountability Journal, 8 (2), 78-101.

Guthrie, J. \& Parker, L. (1989). Corporate social reporting : a rebuttal of legitimacy theory. Accounting and Business Research, 19 (76), 343-352.

Hogner, R.H. (1982). Corporate social reporting: eight decades of development at US Steel. Research in corporate performance and policy, 243-250.

Igalens, J. (2004). Comment évaluer les rapports de développement durable ?. Revue Française de Gestion, 30, 151-167.

Igalens, J. (2006). L'analyse du discours de la responsabilité sociale de l'entreprise à travers les rapports annuels de développement durable d'entreprises françaises du CAC 40. Cahier de recherche 2006-173, LIRHE, Université de Toulouse.

Jenkins, H. \& Yakovleva, N. (2006). Corporate social responsibility in the mining industry: exploring trends in social and environmental disclosure. Journal of cleaner production, 14, 271-284.

Jupe, R. (2005). Disclosures in corporate environmental reports, a test of legitimacy theory. Working paper, 91, University of Kent.

Lentz, A. \& Tschirgi, H. (1963). The ethical content of annual reports. The Journal of Business, 16 (4), Octobre, 387-393. 
Maignan, I. \& Ralston, D. (2002). Corporate social responsibility in Europe and the US: Insights from businesses' self-presentations. Journal of International Business Studies, 497-514.

Mikol, A. (2001). Les informations environnementales diffusées par les grandes entreprises françaises de 1992 à 1999 : résultats d'une étude empirique illustrant la théorie des parties prenantes. Actes du 22e Congrès de l'Association Française de Comptabilité, 2001, 17-19 mai, Metz.

Milne, M. J. \& Adler, R. W. (1999). Exploring the reliability of social and environmental disclosures content analysis. Accounting, Auditing and Accountability Journal, 12 (2), 237-256.

Neu, D., Warsame, H. \& Pedwell, K. (1998). Managing public impressions: environmental disclosures in annual reports. Accounting, Organizations and Society, 23 (33), 265-282.

Ortiz, Martinez, E. \& Crowthern, D. (2008). Is disclosure the right way to comply with stakeholders? the Shell Case. Business Ethics : A European Review, 17 (1), January, 13-22.

Oxibar, B. (2003). La diffusion d'information sociétale dans les rapports annuels et sur les sites Internet des entreprises françaises. Thèse de doctorat en Sciences de gestion.

Oxibar, B. (2005). La diffusion d'information sociétale : outil de mesure et déterminants. Une comparaison multi-supports. 26 éme congrès de l'AFC, 11-13 mai, Lille.

Oxibar, B. (2009). Communication sociétale - Théories et pratiques. L'Harmattan, Collection Entreprises et Management.

Powell, W. \& DiMaggio, P. (Eds.) (1991). The new institutionalism in organizational analysis. Chicago: University of Chicago Press.

Suchman, M. (1995). Managing legitimacy: strategic and institutional approaches. Academy of Management Review, 20 (3), 571-610.

Swanson, D. (1995). Addressing a theoretical problem by reorienting the corporate social performance model. Academy of Management Review, 20 (1), 43-64.

Tilt, C.A. (1994). The influence of external pressure groups on corporate social disclosure: Some empirical evidence. Accounting, Auditing and Accountability Journal, 7 (4), 47-72.

Unerman, J. (2000). Methodological issues - Reflections on quantification in corporate social reporting content analysis. Accounting, Auditing and Accountability Journal, 13 (5), 667-680.

Wilmshurst, T. \& Frost, G. (2000). Corporate environmental reporting : a test of legitimacy theory. Accounting, Auditing and Accountability Journal, 13 (1), 10-26.

Wood, D.J. (1991). Corporate social performance revisited. Academy of Management Review, 16 (4), 691-718. 